\title{
ASYMPTOTIC BEHAVIOUR OF A NONAUTONOMOUS COOPERATIVE SYSTEM
}

\author{
ZHU HONGLIANG ${ }^{1}$ and CUI JINGAN ${ }^{2}$
}

(Received 13 May 1996; revised 16 October 1999)

\begin{abstract}
This paper considers a nonautonomous cooperative system, in which all the parameters are time-dependent and asymptotically approach periodic functions. We prove that under some appropriate conditions any positive solutions of the system asymptotically approach the unique positive periodic solution of the corresponding periodic system.
\end{abstract}

\section{Introduction}

We consider a system of two cooperative species [1]:

$$
\left\{\begin{array}{l}
\dot{u}(t)=r_{1}(t) u(t)\left[1-\frac{u(t)}{a_{1}(t)+b_{1}(t) v(t)}-c_{1}(t) u(t)\right] \\
\dot{v}(t)=r_{2}(t) v(t)\left[1-\frac{v(t)}{a_{2}(t)+b_{2}(t) u(t)}-c_{2}(t) v(t)\right]
\end{array},\right.
$$

where $u(t)$ and $v(t)$ are the densities of the species at time $t$, and the functions $r_{i}(t), a_{i}(t), b_{i}(t), c_{i}(t),(i=1,2)$, are continuous and bounded above and below by positive constants on the half interval $[0, \infty)$. If all the parameters of system $(*)$ are positive constants, this is the simple system that $R$. M. May suggested to describe a pair of mutualists $([2,5])$. In this model, the dynamics of each species follow logistic equations, but the existence of one species can make the capacity of another increase. Now our interest lies in the existence of the positive periodic orbit which is globally asymptotically stable. At first, we define $g_{L}=\inf \{g(t) \mid t \in[0, \infty)\}$ and $g_{M}=\sup \{g(t) \mid t \in[0, \infty)\}$ for a continuous and bounded function $g(t)$. In [3], with $k_{i}=1 / c_{i L}, \delta_{i}=1 /\left(c_{i M}+1 / a_{i L}\right),(i=1,2)$ and $(u(t), v(t))$ being any solution of $(*)$

\footnotetext{
${ }^{1}$ Department of Mathematics, Nanjing University, Nanjing 210093, P. R. China.

${ }^{2}$ School of Math. and Computer Sci., Nanjing Normal University, Nanjing 210097, P. R. China.

(C) Australian Mathematical Society 2001, Serial-fee code 0334-2700/01
} 
such that $u(0)>0, v(0)>0$, it was found that $\delta_{1}<u(t)<k_{1}, \delta_{2}<v(t)<k_{2}$, for sufficiently large $t$. Moreover, if the condition

$$
\begin{gathered}
r_{1 L} r_{2 L}\left(\frac{1}{a_{1 M}+b_{1 M} k_{2}}+c_{1 L}\right)\left(\frac{1}{a_{2 M}+b_{2 M} k_{1}}+c_{2 L}\right) \\
>\frac{r_{1 M} r_{2 M} b_{1 M} b_{2 M} k_{1} k_{2}}{\left(a_{1 L}+b_{1 L} \delta_{2}\right)^{2}\left(a_{2 L}+b_{2 L} \delta_{1}\right)^{2}}
\end{gathered}
$$

holds, then the system (*) has a globally asymptotically stable solution. Furthermore, when the functions $r_{i}(t), a_{i}(t), b_{i}(t)$ and $c_{i}(t)(i=1,2)$ are positive and $T$-periodic, the system $(*)$ has a globally asymptotically stable positive periodic solution $\gamma_{0}(t)=$ $\left(u_{0}(t), v_{0}(t)\right)$, and $\delta_{1}<u_{0}(t)<k_{1}, \delta_{2}<v_{0}(t)<k_{2}$, for $t \geq 0$. But because of the influence of all kinds of environmental conditions, the parameters of the system $(*)$ may not oscillate in accordance with periodic time exactly, but can asymptotically approach periodic functions. So it is important to study the system (*) under these circumstances. In [4], Freedman et al. studied the asymptotic behaviour of a singlespecies model $\dot{x}=x g(x, k(t))$, where $k(t)$ is asymptotic to a periodic function $\tilde{k}(t)$. In [6], by the comparison theorem and continuity theorems of solutions of differential equations, Peng and Chen discussed the asymptotic behaviour of two-species LotkaVolterra competition models, in which all the parameters asymptotically approach periodic functions.

In this paper, we study the asymptotic behaviour of a nonautonomous cooperative system $(*)$. First, we transform the system $(*)$ into the following set of differential equations, where the parameters are still represented by $r_{i}, a_{i}, b_{i}$ and $c_{i}$ :

$$
\left\{\begin{array}{l}
\dot{u}(t)=u(t)\left[r_{1}(t)-\frac{u(t)}{a_{1}(t)+b_{1}(t) v(t)}-c_{1}(t) u(t)\right] \\
\dot{v}(t)=v(t)\left[r_{2}(t)-\frac{v(t)}{a_{2}(t)+b_{2}(t) u(t)}-c_{2}(t) v(t)\right] .
\end{array}\right.
$$

Now let

$$
k_{i}^{ \pm \epsilon}=\frac{r_{i M} \pm \epsilon}{c_{i L} \mp \epsilon}, \quad \delta_{i}^{ \pm \epsilon}=\frac{r_{i L} \pm \epsilon}{1 /\left(a_{i L} \pm \epsilon\right)+c_{i M} \mp \epsilon}, \quad(i=1,2) .
$$

We are going to show that if $r_{i}(t), a_{i}(t), b_{i}(t), c_{i}(t)(i=1,2)$ are asymptotic to periodic functions $\tilde{r}_{i}(t), \tilde{a}_{i}(t), \tilde{b}_{i}(t), \tilde{c}_{i}(t)$, respectively, and if there exists a sufficiently small $\epsilon_{0}>0$ such that the condition

$$
\begin{gathered}
{\left[\frac{1}{\left.\left(a_{1 M}+\epsilon_{0}\right)+\left(b_{1 M}+\epsilon_{0}\right) k_{2}^{+\epsilon_{0}}+\left(c_{1 L}-\epsilon_{0}\right)\right]\left[\frac{1}{\left(a_{2 M}+\epsilon_{0}\right)+\left(b_{2 M}+\epsilon_{0}\right) k_{1}^{+\epsilon_{0}}}+\left(c_{2 L}-\epsilon_{0}\right)\right]}\right.} \\
>\frac{\left(b_{1 M}+\epsilon_{0}\right)\left(b_{2 M}+\epsilon_{0}\right) k_{1}^{-\epsilon_{0}} k_{2}^{-\epsilon_{0}}}{\left[\left(a_{1 L}-\epsilon_{0}\right)+\left(b_{1 L}-\epsilon_{0}\right) \delta_{2}^{-\epsilon_{0}}\right]^{2}\left[\left(a_{2 L}-\epsilon_{0}\right)+\left(b_{2 L}-\epsilon_{0}\right) \delta_{1}^{\left.-\epsilon_{0}\right]^{2}}\right.}
\end{gathered}
$$

holds, then any positive solution of the system asymptotically approaches a positive periodic solution of the corresponding periodic system. 


\section{Some definitions and lemmas}

Before we prove the main result of this paper, it is necessary to give some definitions and lemmas.

Definition 2.1. Let functions $\varphi(t), \psi(t):[0,+\infty) \rightarrow R$. The function $\varphi(t)$ is said to approach $\psi(t)$ asymptotically, if

$$
|\varphi(t)-\psi(t)| \rightarrow 0, \quad t \rightarrow \infty .
$$

Let us denote this by $\varphi(t) \sim \psi(t)$.

DEFINITION 2.2. Let $(u(t), v(t)),(\tilde{u}(t), \tilde{v}(t)):[0,+\infty) \rightarrow R^{2}$. Then $(u(t), v(t))$ is said to approach $(\tilde{u}(t), \tilde{v}(t))$ asymptotically, if

$$
u(t) \sim \tilde{u}(t) \quad \text { and } \quad v(t) \sim \tilde{v}(t),
$$

that is, $(u(t), v(t)) \sim(\tilde{u}(t), \tilde{v}(t))$.

LEMMA 2.1. The set $R_{+}^{2}=\{(u, v) \mid u>0, v>0\}$ is a positive invariant set of system (1.1).

PROOF. Let $(u(t), v(t))$ be a positive solution of the logistic system

$$
\left\{\begin{array}{l}
\dot{u}(t)=u(t)\left[r_{1}(t)-\left(\frac{1}{a_{1}(t)}+c_{1}(t)\right) u(t)\right] \\
\dot{v}(t)=v(t)\left[r_{2}(t)-\left(\frac{1}{a_{2}(t)}+c_{2}(t)\right) v(t)\right] .
\end{array}\right.
$$

It is easy to show that $(u(t), 0)$ and $(0, v(t))$ are solutions of system (1.1). So it follows from the uniqueness theorem [1] that if $(u(t), v(t))$ is a solution of system (1.1) with $u(0)>0$ and $v(0)>0$, then for all $t \geq 0,(u(t), v(t)) \in R_{+}^{2}=\{(u, v) \mid u>0, v>$ $0\}$, and the proof is complete.

For system (1.1), based on the discussion of system (*) in [3], we can obtain the following result.

LEMMA 2.2. Suppose the functions $r_{i}(t), a_{i}(t), b_{i}(t)$ and $c_{i}(t)(i=1,2)$ are positive and T-periodic. Let $k_{i}=r_{i M} / c_{i L}, \delta_{i}=r_{i L} /\left(1 / a_{i L}+c_{i M}\right)$ and if

$$
\left(\frac{1}{a_{1 M}+b_{1 M} k_{2}}+c_{1 L}\right)\left(\frac{1}{a_{2 M}+b_{2 M} k_{1}}+c_{2 L}\right)>\frac{b_{1 M} b_{2 M} k_{1} k_{2}}{\left(a_{1 L}+b_{1 L} \delta_{2}\right)^{2}\left(a_{2 L}+b_{2 L} \delta_{1}\right)^{2}},
$$

then system (1.1) has a globally asymptotically stable positive periodic solution $\gamma_{0}(t)=\left(u_{0}(t), v_{0}(t)\right)$ such that $\delta_{1}<u_{0}(t)<k_{1}$ and $\delta_{2}<v_{0}(t)<k_{2}$ for all $t \geq 0$. 
Consider the systems

and

$$
\left\{\begin{array}{l}
\dot{x_{1}}(t)=x_{1}(t)\left[R_{1}(t)-\frac{x_{1}(t)}{A_{1}(t)+B_{1}(t) x_{2}(t)}-C_{1}(t) x_{1}(t)\right] \\
\dot{x_{2}}(t)=x_{2}(t)\left[R_{2}(t)-\frac{x_{1}(t)}{A_{2}(t)+B_{2}(t) x_{1}(t)}-C_{2}(t) x_{2}(t)\right]
\end{array}\right.
$$

$$
\left\{\begin{array}{l}
\dot{y_{1}}(t)=y_{1}(t)\left[R_{1}^{\prime}(t)-\frac{y_{1}(t)}{A_{1}^{\prime}(t)+B_{1}^{\prime}(t) y_{2}(t)}-C_{1}^{\prime}(t) y_{1}(t)\right] \\
\dot{y_{2}}(t)=y_{2}(t)\left[R_{2}^{\prime}(t)-\frac{y_{2}(t)}{A_{2}^{\prime}(t)+B_{2}^{\prime}(t) y_{1}(t)}-C_{2}^{\prime}(t) y_{2}(t)\right]
\end{array}\right.
$$

We have the following comparison result.

LEMMA 2.3. Assume that $\left(x_{1}(t), x_{2}(t)\right)$ and $\left(y_{1}(t), y_{2}(t)\right)$ are solutions of systems (2.1) and (2.2) respectively, satisfying $x_{i}\left(t_{0}\right)=y_{i}\left(t_{0}\right)>0(i=1,2)$ for some number $t_{0}>0$. If the inequalities $R_{i}(t)>R_{i}^{\prime}(t), A_{i}(t)>A_{i}^{\prime}(t), B_{i}(t)>B_{i}^{\prime}(t)$ and $C_{i}(t)<C_{i}^{\prime}(t)(i=1,2)$ hold for all $t>t_{0}$, then

$$
x_{i}(t)>y_{i}(t) \quad(i=1,2)
$$

for all $t>t_{0}$.

ProOF. Under our assumptions, we have

$$
\begin{aligned}
\dot{x}_{1}\left(t_{0}\right)-\dot{y}_{1}\left(t_{0}\right)= & x_{1}\left(t_{0}\right)\left[R_{1}\left(t_{0}\right)-\frac{x_{1}\left(t_{0}\right)}{A_{1}\left(t_{0}\right)+B_{1}\left(t_{0}\right) x_{2}\left(t_{0}\right)}-C_{1}\left(t_{0}\right) x_{1}\left(t_{0}\right)\right] \\
& -y_{1}\left(t_{0}\right)\left[R_{1}^{\prime}\left(t_{0}\right)-\frac{y_{1}\left(t_{0}\right)}{A_{1}^{\prime}\left(t_{0}\right)+B_{1}^{\prime}\left(t_{0}\right) y_{2}\left(t_{0}\right)}-C_{1}^{\prime}\left(t_{0}\right) y_{1}\left(t_{0}\right)\right]>0
\end{aligned}
$$

and $\dot{x_{2}}\left(t_{0}\right)-\dot{y_{2}}\left(t_{0}\right)>0$. The inequalities

$$
x_{1}(t)>y_{1}(t) \text { and } x_{2}(t)>y_{2}(t)
$$

will therefore hold for $t-t_{0}>0$ sufficiently small. Assume that (2.3) does not hold for all $t>t_{0}$. Then there exists $t_{1}>t_{0}$ such that $x_{i}(t)>y_{i}(t)$, for all $t \in\left(t_{0}, t_{1}\right)$, and either: (i) $x_{1}\left(t_{1}\right)=y_{1}\left(t_{1}\right)$ or (ii) $x_{2}\left(t_{1}\right)=y_{2}\left(t_{1}\right)$. Suppose that (i) holds. By continuity we must have $x_{2}\left(t_{1}\right) \geq y_{2}\left(t_{1}\right)$, and by invariance of the open first quadrant, $x_{1}\left(t_{1}\right)=y_{1}\left(t_{1}\right)>0$. Since $x_{1}(t)-y_{1}(t)>0$ on $\left(t_{0}, t_{1}\right)$, we have $\dot{x_{1}}\left(t_{1}\right)-\dot{y_{1}}\left(t_{1}\right) \leq 0$. On the other hand, from (2.1) and (2.2), we have that

$$
\begin{aligned}
\dot{x}_{1}\left(t_{1}\right)-\dot{y}_{1}\left(t_{1}\right)= & x_{1}\left(t_{1}\right)\left[R_{1}\left(t_{1}\right)-\frac{x_{1}\left(t_{1}\right)}{A_{1}\left(t_{1}\right)+B_{1}\left(t_{1}\right) x_{2}\left(t_{1}\right)}-C_{1}\left(t_{1}\right) x_{1}\left(t_{1}\right)\right] \\
& -y_{1}\left(t_{1}\right)\left[R_{1}^{\prime}\left(t_{1}\right)-\frac{y_{1}\left(t_{1}\right)}{A_{1}^{\prime}\left(t_{1}\right)+B_{1}^{\prime}\left(t_{1}\right) y_{2}\left(t_{1}\right)}-C_{1}^{\prime}\left(t_{1}\right) y_{1}\left(t_{1}\right)\right]>0 .
\end{aligned}
$$


This contradiction shows that case (i) does not hold, and a similar argument proves the same for case (ii). So

$$
x_{i}(t)>y_{i}(t) \quad(i=1,2)
$$

for all $t \in\left(t_{0},+\infty\right)$. And the proof is complete.

We now give some assumptions. We suppose that $r_{i}(t) \sim \tilde{r}_{i}(t), a_{i}(t) \sim \tilde{a}_{i}(t)$, $b_{i}(t) \sim \tilde{b}_{i}(t)$ and $c_{i}(t) \sim \tilde{c}_{i}(t)$, where functions $\tilde{r}_{i}(t), \tilde{a}_{i}(t), \tilde{b}_{i}(t)$ and $\tilde{c}_{i}(t)$ are positive and $T$-periodic. So for all $\epsilon \in\left(0, \epsilon_{0}\right)$, there exists $t_{\epsilon}>0$ such that

$$
\left.\begin{array}{cc}
\left|r_{i}(t)-\tilde{r}_{i}(t)\right|<\epsilon, & \left|a_{i}(t)-\tilde{a}_{i}(t)\right|<\epsilon \\
\left|b_{i}(t)-\tilde{b}_{i}(t)\right|<\epsilon, & \left|c_{i}(t)-\tilde{c}_{i}(t)\right|<\epsilon
\end{array}\right\}
$$

hold, as $t>t_{\epsilon}$.

Following Peng and Chen [6], we construct the following three systems

$$
\begin{aligned}
& \left\{\begin{array}{l}
\dot{u}(t)=u(t)\left[\tilde{r}_{1}(t)-\frac{u(t)}{\tilde{a}_{1}(t)+\tilde{b}_{1}(t) v(t)}-\tilde{c}_{1}(t) u(t)\right] \\
\dot{v}(t)=v(t)\left[\tilde{r}_{2}(t)-\frac{v(t)}{\tilde{a}_{2}(t)+\tilde{b}_{2}(t) u(t)}-\tilde{c}_{2}(t) v(t)\right]
\end{array}\right. \\
& \left\{\begin{array}{l}
\dot{u}(t)=u(t)\left[\left(\tilde{r}_{1}(t)+\epsilon\right)-\frac{u(t)}{\left(\tilde{a}_{1}(t)+\epsilon\right)+\left(\tilde{b}_{1}(t)+\epsilon\right) v(t)}-\left(\tilde{c}_{1}(t)-\epsilon\right) u(t)\right] \\
\dot{v}(t)=v(t)\left[\left(\tilde{r}_{2}(t)+\epsilon\right)-\frac{v(t)}{\left(\tilde{a}_{2}(t)+\epsilon\right)+\left(\tilde{b}_{2}(t)+\epsilon\right) u(t)}-\left(\tilde{c}_{2}(t)-\epsilon\right) v(t)\right]
\end{array}\right.
\end{aligned}
$$

and

$$
\left\{\begin{array}{l}
\dot{u}(t)=u(t)\left[\left(\tilde{r}_{1}(t)-\epsilon\right)-\frac{u(t)}{\left(\tilde{a}_{1}(t)-\epsilon\right)+\left(\tilde{b}_{1}(t)-\epsilon\right) v(t)}-\left(\tilde{c}_{1}(t)+\epsilon\right) u(t)\right] \\
\dot{v}(t)=v(t)\left[\left(\tilde{r}_{2}(t)-\epsilon\right)-\frac{v(t)}{\left(\tilde{a}_{2}(t)-\epsilon\right)+\left(\tilde{b}_{2}(t)-\epsilon\right) u(t)}-\left(\tilde{c}_{2}(t)+\epsilon\right) v(t)\right] .
\end{array}\right.
$$

From (2.4) and Lemma 2.3, we get the following result.

LEMMA 2.4. Assume that $(u(t), v(t)),\left(u^{+\epsilon}(t), v^{+\epsilon}(t)\right)$ and $\left(u^{-\epsilon}(t), v^{-\epsilon}(t)\right)$ are solutions of systems (1.1), (2.6) and (2.7) respectively, satisfying $u\left(t_{\epsilon}\right)=u^{-\epsilon}\left(t_{\epsilon}\right)=$ $u^{+\epsilon}\left(t_{\epsilon}\right)>0, v\left(t_{\epsilon}\right)=v^{-\epsilon}\left(t_{\epsilon}\right)=v^{+\epsilon}\left(t_{\epsilon}\right)>0$. Then

$$
u^{-\epsilon}(t)<u(t)<u^{+\epsilon}(t), \quad v^{-\epsilon}(t)<v(t)<v^{+\epsilon}(t)
$$

for all $t>t_{\epsilon}$. 
ProOF. It follows from (2.4) that

$$
\left.\begin{array}{rl}
\tilde{r}_{i}(t)-\epsilon<r_{i}(t)<\tilde{r}_{i}(t)+\epsilon, & \tilde{a}_{i}(t)-\epsilon<a_{i}(t)<\tilde{a}_{i}(t)+\epsilon \\
\tilde{b}_{i}(t)-\epsilon<b_{i}(t)<\tilde{b}_{i}(t)+\epsilon, & \tilde{c}_{i}(t)-\epsilon<c_{i}(t)<\tilde{c}_{i}(t)+\epsilon
\end{array}\right\}
$$

for all $t>t_{\epsilon}$. For systems (1.1), (2.6) and (2.7), by Lemma 2.3, we have

$$
u^{-\epsilon}(t)<u(t)<u^{+\epsilon}(t), \quad v^{-\epsilon}(t)<v(t)<v^{+\epsilon}(t)
$$

for all $t>t_{\epsilon}$. This proves the lemma.

The following results describe the existence of globally asymptotically stable periodic solutions of systems (2.5), (2.6) and (2.7).

LEMMA 2.5. If condition (1.2) holds, then there exist positive T-periodic solutions $(\tilde{u}(t), \tilde{v}(t)),\left(u^{+\epsilon}(t), v^{+\epsilon}(t)\right)$ and $\left(u^{-\epsilon}(t), v^{-\epsilon}(t)\right)$ of systems (2.5), (2.6) and (2.7) respectively, which are all globally asymptotically stable. Moreover,

$$
\left.\begin{array}{lll}
\tilde{\delta}_{1}<\tilde{u}(t)<\tilde{k}_{1}, & \tilde{\delta}_{2}<\tilde{v}(t)<\tilde{k}_{2}, & \tilde{\delta}_{1}^{+\epsilon}<u^{+\epsilon}(t)<\tilde{k}_{1}^{+\epsilon} \\
\tilde{\delta}_{2}^{+\epsilon}<v^{+\epsilon}(t)<\tilde{k}_{2}^{+\epsilon}, & \tilde{\delta}_{1}^{-\epsilon}<u^{-\epsilon}(t)<\tilde{k}_{1}^{-\epsilon}, & \tilde{\delta}_{2}^{-\epsilon}<v^{-\epsilon}(t)<\tilde{k}_{2}^{-\epsilon}
\end{array}\right\},
$$

where

$$
\begin{gathered}
\tilde{k}_{i}=\frac{\tilde{r}_{i M}}{\tilde{c}_{i L}}, \quad \tilde{\delta}_{i}=\frac{\tilde{r}_{i L}}{1 / \tilde{a}_{i L}+\tilde{c}_{i M}}, \quad \tilde{k}_{i}^{ \pm \epsilon}=\frac{\tilde{r}_{i M} \pm \epsilon}{\tilde{c}_{i L} \mp \epsilon} \quad \text { and } \\
\tilde{\delta}_{i}^{ \pm \epsilon}=\frac{\tilde{r}_{i L} \pm \epsilon}{1 /\left(\tilde{a}_{i L} \pm \epsilon\right)+\tilde{c}_{i M} \mp \epsilon} \quad(i=1,2)
\end{gathered}
$$

ProOF. Under condition (1.2) and from (2.9), for all $\epsilon \in\left(0, \epsilon_{0}\right)$ it is easy to show that

$$
\begin{gathered}
\left(\frac{1}{\tilde{a}_{1 M}+\tilde{b}_{1 M} \tilde{k}_{2}}+\tilde{c}_{1 L}\right)\left(\frac{1}{\tilde{a}_{2 M}+\tilde{b}_{2 M} \tilde{k}_{1}}+\tilde{c}_{2 L}\right)>\frac{\tilde{b}_{1 M} \tilde{b}_{2 M} \tilde{k}_{1} \tilde{k}_{2}}{\left(\tilde{a}_{1 L}+\tilde{b}_{1 L} \tilde{\delta}_{2}\right)^{2}\left(\tilde{a}_{2 L}+\tilde{b}_{2 L} \tilde{\delta}_{1}\right)^{2}} \\
{\left[\frac{1}{\left(\tilde{a}_{1 M}+\epsilon\right)+\left(\tilde{b}_{1 M}+\epsilon\right) \tilde{k}_{2}^{+\epsilon}}+\left(\tilde{c}_{1 L}-\epsilon\right)\right]\left[\frac{1}{\left(\tilde{a}_{2 M}+\epsilon\right)+\left(\tilde{b}_{2 M}+\epsilon\right) \tilde{k}_{1}^{+\epsilon}}+\left(\tilde{c}_{2 L}-\epsilon\right)\right]} \\
>\frac{\left(\tilde{b}_{1 M}+\epsilon\right)\left(\tilde{b}_{2 M}+\epsilon\right) \tilde{k}_{1}^{+\epsilon} \tilde{k}_{2}^{+\epsilon}}{\left[\left(\tilde{a}_{1 L}+\epsilon\right)+\left(\tilde{b}_{1 L}+\epsilon\right) \tilde{\delta}_{2}^{+\epsilon}\right]^{2}\left[\left(\tilde{a}_{2 L}+\epsilon\right)+\left(\tilde{b}_{2 L}+\epsilon\right) \tilde{\delta}_{1}^{+\epsilon}\right]^{2}} \quad \text { and } \\
{\left[\frac{1}{\left(\tilde{a}_{1 M}-\epsilon\right)+\left(\tilde{b}_{1 M}-\epsilon\right) \tilde{k}_{2}^{-\epsilon}}+\left(\tilde{c}_{1 L}+\epsilon\right)\right]\left[\frac{1}{\left(\tilde{a}_{2 M}-\epsilon\right)+\left(\tilde{b}_{2 M}-\epsilon\right) \tilde{k}_{1}^{-\epsilon}}+\left(\tilde{c}_{2 L}+\epsilon\right)\right]} \\
>\frac{\left(\tilde{b}_{1 M}-\epsilon\right)\left(\tilde{b}_{2 M}-\epsilon\right) \tilde{k}_{1}^{-\epsilon} \tilde{k}_{2}^{-\epsilon}}{\left[\left(\tilde{a}_{1 L}-\epsilon\right)+\left(\tilde{b}_{1 L}-\epsilon\right) \tilde{\delta}_{2}^{-\epsilon}\right]^{2}\left[\left(\tilde{a}_{2 L}-\epsilon\right)+\left(\tilde{b}_{2 L}-\epsilon\right) \tilde{\delta}_{1}^{-\epsilon}\right]^{2}} .
\end{gathered}
$$


According to Lemma 2.2, the above three inequalities guarantee the existence of positive $T$-periodic solutions of systems (2.5), (2.6) and (2.7), which are all globally asymptotically stable, and (2.10) holds true. This proves the lemma.

From this lemma, we are able to show that there exists $\epsilon_{1}>0$ such that intervals $\left[\delta_{i}^{+\epsilon}, k_{i}^{+\epsilon}\right]$ and $\left[\delta_{i}^{-\epsilon}, k_{i}^{-\epsilon}\right](i=1,2)$ for all $\epsilon \in\left(0, \epsilon_{1}\right)$, are all bounded sets. We now present a lemma which is quoted from [6] and so we omit the proof.

LEMMA 2.6. Assume that $(\tilde{u}(t), \tilde{v}(t)),\left(u^{+\epsilon}(t), v^{+\epsilon}(t)\right)$ and $\left(u^{-\epsilon}(t), v^{-\epsilon}(t)\right)$ are the positive T-periodic solutions described in Lemma 2.5. Then there exists $\alpha>0$ such that $\left(u^{-\epsilon}, v^{-\epsilon}\right) \sim(\tilde{u}, \tilde{v})$ and $\left(u^{+\epsilon}, v^{+\epsilon}\right) \sim(\tilde{u}, \tilde{v})$ for all $0<\epsilon<\alpha$.

\section{Main result}

With the above lemmas as a prerequisite, we now give the main result of this paper.

THEOREM. Let $\tilde{r}_{i}(t), \tilde{a}_{i}(t), \tilde{b}_{i}(t)$ and $\tilde{c}_{i}(t)$ be continuous, positive and T-periodic functions. If $r_{i}(t) \sim \tilde{r}_{i}(t), a_{i}(t) \sim \tilde{a}_{i}(t), b_{i}(t) \sim \tilde{b}_{i}(t), c_{i}(t) \sim \tilde{c}_{i}(t)$ and the inequality (1.2) holds, then for any positive solution $(u(t), v(t))$ of system (1.1), we have $(u, v) \sim(\tilde{u}, \tilde{v})$, where $(\tilde{u}, \tilde{v})$ is the unique positive T-periodic solution of system (2.5).

ProOF. Suppose $(u(t), v(t))$ is a positive solution of system (1.1), and let $(\tilde{u}(t)$, $\tilde{v}(t)),\left(u^{+\epsilon}(t), v^{+\epsilon}(t)\right)$ and $\left(u^{-\epsilon}(t), v^{-\epsilon}\right)$ be the globally asymptotically stable positive $T$-periodic solutions of constructed systems (2.5), (2.6) and (2.7) respectively. From Lemma 2.6, for every $\eta>0$, there exists $\alpha>0$ such that

$$
\left|u^{ \pm \epsilon}(t)-\tilde{u}(t)\right|<\eta \quad \text { and } \quad\left|v^{ \pm \epsilon}(t)-\tilde{v}(t)\right|<\eta
$$

for $0<\epsilon<\alpha$. Since $r_{i}(t) \sim \tilde{r}_{i}(t), a_{i}(t) \sim \tilde{a}_{i}(t), b_{i}(t) \sim \tilde{b}_{i}(t)$ and $c_{i}(t) \sim \tilde{c}_{i}(t)$, there exists $t^{\prime}>0$ such that (2.4) holds for all $t \geq t^{\prime}$. Furthermore, let $\left(u^{+\epsilon}(t), v^{+\epsilon}(t)\right)$ and $\left(u^{-\epsilon}(t), v^{-\epsilon}\right)$ satisfy

$$
u^{+\epsilon}\left(t^{\prime}\right)=u^{-\epsilon}\left(t^{\prime}\right)=u\left(t^{\prime}\right) \quad \text { and } \quad v^{+\epsilon}\left(t^{\prime}\right)=v^{-\epsilon}\left(t^{\prime}\right)=v\left(t^{\prime}\right)
$$

From Lemma (2.4), we have

$$
u^{-\epsilon}(t)<u(t)<u^{+\epsilon}(t) \text { and } v^{-\epsilon}(t)<v(t)<v^{+\epsilon}(t)
$$

for all $t>t^{\prime}$. And from Lemma 2.6, we have

$$
\left(u^{-\epsilon}, v^{-\epsilon}\right) \sim(\tilde{u}, \tilde{v}) \text { and }\left(u^{+\epsilon}, v^{+\epsilon}\right) \sim(\tilde{u}, \tilde{v}) .
$$

So according to (A) and (B), we can draw the conclusion that $(u, v) \sim(\tilde{u}, \tilde{v})$. This completes the proof of the theorem. 
As an example, we consider the cooperative system given by

$$
\left\{\begin{aligned}
\dot{u}(t)= & u(t)\left\{\frac{4+\cos (t+1 / t)}{2}-\frac{u(t)}{[4+\cos (t+1 / t)]+[2+\cos (t+1 / t)] v(t)}\right. \\
& \left.-\left[6+\frac{\cos (t+1 / t)}{2}\right] u(t)\right\}, \\
\dot{v}(t)= & v(t)\left\{\frac{3+\cos (t+1 / t)}{2}-\frac{v(t)}{[3+\cos (t+1 / t)]+[2+\cos (t+1 / t)] u(t)}\right. \\
& -[5+\cos (t+1 / t)] v(t)\} .
\end{aligned}\right.
$$

In fact, choosing an arbitrary $\epsilon_{0} \in(0,1]$, we can verify that (1.2) holds and that this system satisfies all conditions in the theorem, so any positive solution of the system must asymptotically approach the unique positive periodic solution of the corresponding periodic system

$$
\left\{\begin{array}{l}
\dot{u}(t)=u(t)\left[\frac{4+\cos t}{2}-\frac{u(t)}{(4+\cos t)+(2+\cos t) v(t)}-\left(6+\frac{\cos t}{2}\right) u(t)\right], \\
\dot{v}(t)=v(t)\left[\frac{3+\cos t}{2}-\frac{v(t)}{(3+\cos t)+(2+\cos t) u(t)}-(5+\cos t) v(t)\right] .
\end{array}\right.
$$

\section{Acknowledgement}

This research was supported by the NNSF of China.

\section{References}

[1] V. I. Arnold, Geometrical methods in the theory of ordinary differential equations (Springer, 1983).

[2] L. S. Chen, Models and Research Methods of Mathematical Ecology, (in Chinese) (Science Press, Beijing, 1988).

[3] J. A. Cui and L. S. Chen, "Global asymptotic stability in a nonautonomous cooperative system", Systems Sci. Math. Sci. 6 (1993) 44-51.

[4] H. I. Freedman, V. Sree Hari Rao and J. W.-H. So, "Asymptotic behavior of a time-dependent single-species model", Analysis 9 (1989) 217-223.

[5] R. M. May, Theoretical ecology, principles and applications (Sounders, 1976).

[6] Q. L. Peng and L. S. Chen, "Asymptotic behavior of the nonautonomous two-species Lotka-Volterra competition models", Comput. Math. Appl. 27 (1994) 53-60. 